\title{
Discovery of two diacetylene glycosides as human uridine-cytidine kinase 2 inhibitors: an in silico approach
}

\author{
Magdi A. Mohamed ${ }^{1 *}$, Amina I. Dirar ${ }^{1,2}$, Sami Hamdoun ${ }^{3}$ \\ ${ }^{1}$ Department of Pharmaceutical Chemistry, Faculty of Pharmacy, University of Khartoum, Sudan. ${ }^{2}$ Department of Phytochemistry and Plant Sciences, \\ Faculty of Pharmacy, National University, Sudan. ${ }^{3}$ Department of Pharmaceutics, Faculty of Pharmacy, University of Khartoum, Sudan.
}

\section{ARTICLE INFO \\ Article history: \\ Received on: 05/06/2016 \\ Revised on: 18/07/2016 \\ Accepted on: 14/08/2016 \\ Available online: 29/11/2016}

Key words:

Ligusticopsis wallichiana

(DC), bhutkesoside A,

bhutkesoside $\mathrm{B}$, in silico

pharmacokinetic, uridine-

cytidine kinase 2 , cancer.

\begin{abstract}
Natural products continue today, in a never-ending quest, to provide new lead compounds. Yet, the path to a marketed drug involves a long and exhaustive journey through basic research. Interestingly, the use of in silicobased research has reduced the cost and time needed to bring a drug to the market. In this context, several chemoinformatics studies were conducted for two new diacetylene glycosides, namely Bhutkesoside A and Bhutkesoside B which were isolated from Ligusticopsis wallichiana (DC) (Apiaceae). In silico target prediction and molecular docking revealed that the two compounds are potential uridine-cytidine kinase 2 (UCK2) inhibitors. Thus, they could be envisioned as new leads for cancer associated with UCK2 over expression. To assess the druggability of these compounds, their physicochemical properties and ADMET profiles were studied using several integrated web based in silico tools.
\end{abstract}

\section{INTRODUCTION}

Drug discovery and development is an iterative process which begins with the identification of lead compounds (Neamati and Barchi, 2002). In this context, the conventional approach is challenging, time consuming, expensive and requires consideration of many aspects that have negative impact on pharmaceutical industries (Earm and Earm, 2014). On the other hand, the use of computational technology, so-called in silico, in drug discovery and development has now surpassed the conventional approach. The in silico approach estimates potential biological activity and druggability of new leads without having to undergo the costly and tedious wet conventional experiments. Thus, the in silico approach facilitates

* Corresponding Author

Magdi A. Mohamed, Department of Pharmaceutical Chemistry,

Faculty of Pharmacy, University of Khartoum, Sudan.

Email: :mawadalla@uofk.edu drug discovery efficiently where the target protein, and thus biological activity, could be predicted at early stage expediting the time required for the discovery process (Sliwoski et al., 2014). In this context, PharmMapper server is used to predict potential protein targets (Liu et al., 2010). The putative binding mode of the ligands with their PharmMapper predicted targets is revealed by molecular docking. This step is crucial in understanding the binding interaction pattern that will pave the way for rational structural modification of tested leads (Mandal et al., 2009). Different web-based servers e.g. Molinspiration (Available at: http://www.molinspiration.com) [Accessed 17 May 2016], AdmetSAR (Cheng et al., 2012) and Metaprint2D (Carlsson et al., 2010) are available for in silico estimation of pharmacokinetic and toxicity profiles of tested leads. Thus, the in silico testing of compounds' pharmacokinetics and toxicity could be done at very early stage so that only candidate compounds meeting required criteria will be submitted to clinical trials (van de Waterbeemd and Gifford, 2003). 
Lead discovery from medicinal plants has attracted researchers' attention. However, the very small quantities of isolated leads is an obstacle for conducting necessary wet experiments before these leads are developed into new drugs (Balunas and Kinghorn, 2005). The medicinal plant Ligusticopsis wallichiana (DC) (Apiaceae) is known to have several curative properties (Padalia et al., 2012; Adhikari et al., 2015). Recently, isolation of two new diacetylene glycosides, namely bhutkesoside A and bhutkesoside B (Figure 1), has been reported (Adhikari et al., 2015) from Ligusticopsis wallichiana (DC) (Apiaceae). To best of our knowledge, biological activity and pharmacokinetic profile have not been reported for bhutkesoside A nor bhutkesoside B since then. We have been involved in estimating druggabilities of phytochemicals using in silico tools (Dirar et al., 2016) and we herein, report the potential uridine-cytidine kinase 2 (UCK2) inhibitory activity, pharmacokinetics and toxicity of bhutkesoside A and bhutkesoside B.

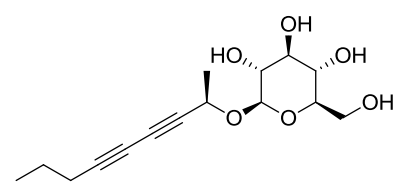

Bhutkesoside A

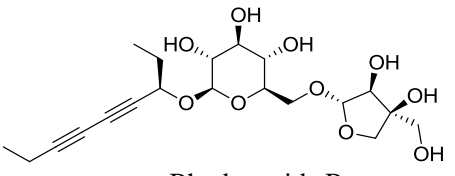

Bhutkesoside B
Fig. 1:

\section{MATERIALS AND METHODS}

\section{General}

The 3D chemical structure of the compounds were prepared using ChemDraw Ultra 12 software and saved as PDB format. When necessary, the PDB format was converted to MDL.sdf or SMILE formats using Open Bable software (O'Boyle et al., 2011). The 3D structure of the protein target was retrieved from protein data bank and optimized manually. The Swiss PDB viewer V.4.1.0. software (Guex and Peitsch, 1997) was used for energy minimization. Molecular docking was performed using Autodock 4.0 software based on Lamarckian Genetic Algorithm (Morris et al., 1998; Morris et al., 2009).

\section{Biological activity}

The potential protein target for the two leads was predicted using PharmMapper server. On the other hand, Molinspiration server was used to predict drug likeness properties of the two leads as G-protein coupled receptor (GPCR) ligands, ion channel modulators (ICM), kinase inhibitors (KI), nuclear receptor ligands (NRL), protease inhibitors (PI) and enzyme inhibitors (EI).

\section{Ligand-protein docking}

Polar hydrogen atoms were added to the protein target and Kollman united atomic charges were computed. All hydrogen atoms were added to the ligands before the Gastiger partial charges were assigned. The co-crystal ligand was removed and the bond orders were checked. The target's grid map was calculated and set to $60 \times 60 \times 60$ points with grid spacing of $0.375 \AA$. The grid box was then allocated properly in the target to include the active residue in the center. The default docking algorithms were set in accordance with standard docking protocol. Finally, ten independent docking runs were carried out for each ligand and results were retrieved as binding energies. Poses that showed lowest binding energies were visualized using MOE (Available at: http://www.chemcomp.com) [Accessed 17 May 2016] and UCSF chimera (Pettersen et al., 2004).

\section{Physicochemical properties}

LogP, topological polar surface area (TPSA) and number of hydrogen bond donors (HBD) and acceptors (HBA) for the two leads were estimated using Molinspiration server.

\section{Pharmacokinetics and toxicity}

Absorption, distribution, metabolism, excretion and toxicity (ADMET) were estimated using AdmetSAR and MetaPrint2D online servers.

\section{RESULTS AND DISCUSSION}

\section{Bioactivity and molecular docking}

PharmMapper server has predicted that uridine-cytidine kinase 2 (UCK2)(PDB: 1UDW) (Suzuki et al., 2004) is the best target, in terms of fit score, for both bhutkesoside $\mathrm{A}$ and bhutkesoside B (5.834 and 6.129, respectively). UCK2 is expressed in various living organisms including humans, animals and microorganisms (Van Rompay et al., 2001). This enzyme plays an important role as a catalyst in the salvage pathway of nucleotide metabolism. It acts by phosphorylating uridine and cytidine to uridine monophosphate (UMP) and cytidine monophosphate (CMP) (Connolly and Duley, 1999). In cells, the activation of UMP and CMP by phosphorylation is essentially required for the formation of uridine triphosphate (UTP) and cytidine triphosphate (CTP). These triphosphate nucleotides are mandatory for DNA and RNA synthesis (Qian et al., 2014; Malami et al., 2016). It has been reported that UCK2 is normally expressed in the placenta. Moreover, UCK2 has been found to be over expressed in certain tumors (Appleby et al., 2005; Zlatopolskiy et al., 2009). Furthermore, the association between UCK2 and testicular germ cell tumors has been supported by the outcomes of genome wide association studies (Murata et al., 2004; Sripayap et al., 2014; Valencia et al., 2014). The selective expression of UCK2 in cancer cells makes it a potential target for cancer chemotherapy (Malami et al., 2016). Having identified UCK2 as a potential target for both bhutkesoside $\mathrm{A}$ and bhutkesoside $\mathrm{B}$, we next turned to study the biochemical interactions of the two leads with UCK2 active site. In this context, molecular docking studies revealed an interesting lignad interaction with UCK2 for both bhutkesoside A and bhutkesoside B (Figure 2) with satisfactory binding energies ( -9.15 and -8.81 $\mathrm{Kcal} / \mathrm{mol}$, respectively). Bhutkesoside A binds the key residues LYS33 and ARG174 via hydrogen bonds. 


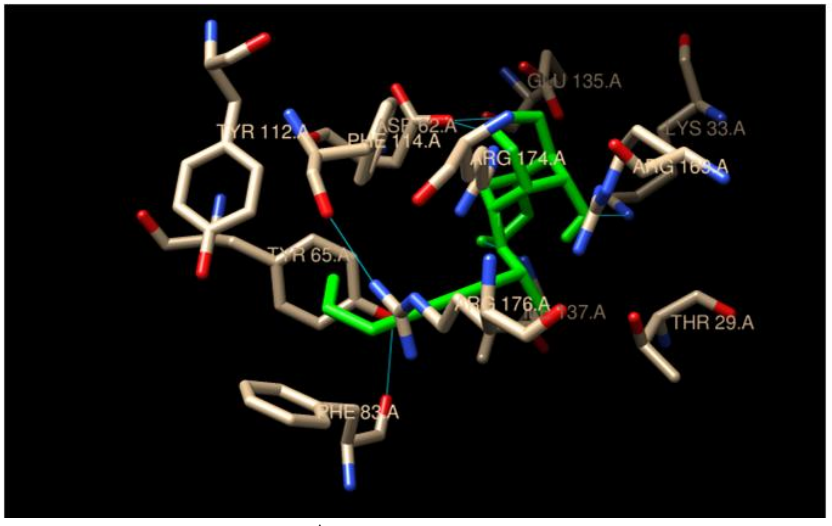

A

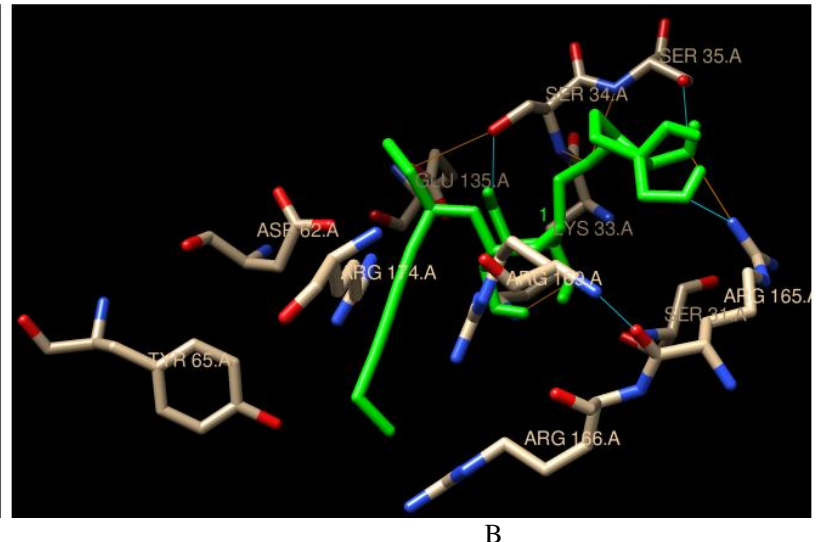

B

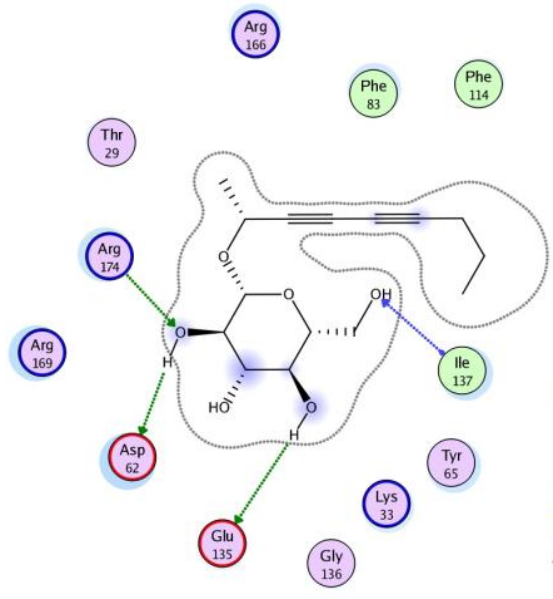

C
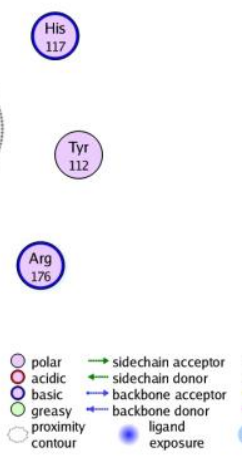
ligand
exposure

Fig. 2: Ligand-UCK2 interactions Bhutkesoside A-UCK2 interactions visualized by (a) Chimera and (c) MOE Bhutkesoside B-UCK2 interactions visualized by (b) Chimera and (d) MOE.
On the other hand, like bhutkesoside A, bhutkesoside B also binds to LYS33 via hydrogen bond. It is worth noting that LYS33 and ARG174 along with ARG169 are the potential candidates to stabilize the anionic charge on the pentacoordinate transition state adopted by ATP upon nucleophilic attack by the $5_{1^{-}}$ oxygen atom of the ribonucleoside at the $\gamma$ phosphate moiety of ATP (Suzuki et al., 2004). A hydrogen bond is also formed between bhutkesoside A and the key residue ASP62 which is the only available catalytic base around the $5_{1}$-hydroxyl group of the ribonucleoside. Base-catalyzed activation of the 5 -hydroxyl group of ribonucleosides facilitates its nucleophilic attack at the $\gamma$ phosphate moiety of ATP (Suzuki et al., 2004; Appleby et al., 2005). It has been shown that ligand binding at both the phosphate donor and acceptor binding sites competitively inhibit UCK2 (Suzuki et al., 2004). Therefore, it is anticipated that in the presence of bhutkesoside A, the carboxyl side chain of ASP62 will be hydrogen bonded to bhutkesoside $A$ and, thus, the $5_{1}$-hydroxyl group of ribonucleosides will not be activated resulting in UCK2 competitive inhibition. Contrary to bhutkesoside A, bhutkesoside B does not bind to ILE137 or PHE114 via hydrogen bond nor hydrophobic interactions. Nevertheless, bhutkesoside B buried itself deep in the ATP binding pocket surrounded by amino acid side chains where $\gamma$ - and $\beta$-phosphate moieties of ATP bind. In this context, bhutkesoside B binds with the key residues GLY32, SER34 and SER35 via hydrogen bonds. Bhutkesoside B is, therefore, estimated to inhibit UCK2 protein by binding to the catalytic active site of ATP, thus inhibiting ATP from binding to its active site in the UCK2 protein. Hydrophobic interactions were also predicted for bhutkesoside A and bhutkesoside B with PHE83 and ALA30, respectively. Interestingly, ALA30, GLY32, SER34 and PHE83 have been reported to be involved in the interaction of UCK2 with known inhibitors (Malami et al., 2016). With their UCK2 competitive inhibition, both bhutkesoside $\mathrm{A}$ and bhutkesoside B could be envisioned as potential leads for cancer associated with over expression of UCK2. Druggability likeliness property of Bhutkesoside A and Bhutkesoside B as GPCR ligands, ion channel modulators (ICM), kinase inhibitors (KI), nuclear receptor ligands (NRL), protease inhibitors (PI) and enzyme inhibitors (EI) were studied, and results were retrieved as bioactivity scores. In this context, scores $>0.00$ indicate high activity, between 0.00 to -0.5 indicate moderate activity and $<-0.5$ indicate inactivity (Dirar et al., 2016). High activities were 
retrieved for both Bhutkesoside A and Bhutkesoside B except for Bhutkesoside A as KI where it was predicted to be moderately active (Table 1). The highest activity for both compounds was associated with EI and this could support their potential inhibition of the UCK2 enzyme.

Table 1: Molinspiration estimated drug likeliness property for each lead.

\begin{tabular}{ccccccc}
\hline Lead & GPCR & ICM & KI & NRL & PI & EI \\
\hline Bhutkesoside A & 0.37 & 0.43 & -0.01 & 0.26 & 0.17 & 0.67 \\
Bhutkesoside B & 0.41 & 0.46 & 0.08 & 0.21 & 0.37 & 0.71 \\
\hline
\end{tabular}

\section{Physicochemical properties, pharmacokinetics and toxicity}

According to Lipinski's rule of five, poor absorption or permeation is more likely when there are more than $5 \mathrm{H}$-bond donors, $10 \mathrm{H}$-bond acceptors, the molecular weight is greater than $500 \mathrm{Da}$ and the calculated $\log \mathrm{P}(\mathrm{C} \log \mathrm{P})$ is greater than 5 (or MlogP>4.15) (Lipinski et al., 2001). Moreover, good bioavailability is more likely for compounds with $\leq 10$ rotatable bonds (nrotb) and total polar surface area (TPSA) of $\leq 140 \AA$ (Veber et al., 2002). Fortunately, Molinspiration server predicts these physicochemical properties without having to conduct wet experiments. To this end, bhutkesoside A was potentially shown to have good absorption and permeation and thus good oral bioavailability where all its predicted physicochemical properties were in agreement with Lipinski's rule of five (Table 2). In contrast, the oral bioavailability of bhutkesoside B is questionable where three parameters, namely, TPSA, HBA and HBD were outranged. However, like Bhutkesoside A, the molecular weight of Bhutkesoside B is less than $500 \mathrm{Da}$, thus, it is easily transported, diffuse and absorbed compared to heavy molecules (Srimai et al., 2013).

Table 2: Molinspiration predicted physicochemical parameters for each lead.

\begin{tabular}{ccccccc}
\hline Lead & $\begin{array}{c}\text { Mi } \\
\log \end{array}$ & TPSA & MW & HBA & HBD & nrotb \\
\hline Bhutkesoside A & 0.97 & 99.38 & 293 & 6 & 4 & 4 \\
Bhutkesoside B & -0.50 & 158.30 & 430 & 10 & 6 & 7 \\
\hline
\end{tabular}

The ADMET properties of Bhutkesoside $\mathrm{A}$ and Bhutkesoside B were calculated using admetSAR. Blood Brain Barrier (BBB) penetration, HIA (Human Intestinal Absorption), human colon carcinoma cell (Caco-2) permeability and AMES test were calculated. Contrary to bhutkesoside B, bhutkesoside A showed a positive result for BBB which indicates its CNS penetrability. Often a parabolic relationship exists between measured lipophilicity and in vivo brain penetration of drugs, where those moderate in lipophilicity often exhibit highest uptake. On the other hand, very polar compounds normally exhibit less lipid solubility that limits BBB penetration (Waterhouse, 2003). Molinspiration server has predicted that bhutkesoside $A$ is moderately lipophilic ( $\log \mathrm{P}=0.97$ ) while bhutkesoside $\mathrm{B}$ is less lipophilic $(\log P=-0.5)$. Such a difference in the lipophilicity could explain the unique BBB penetrability of bhutkesoside A. Both compounds were predicted not to penetrate through Caco-2 cell line nor to have human intestine absorption. It is worth noting that, the permeability coefficients across mono-layers of Caco-2 are commonly used to predict the absorption of orally administered drugs and other xenobiotics (Hubatsch et al., 2005). Consequently, the tested compounds could be of low oral bioavailability and need further structural optimization. Caco-2 model has also been proposed for prediction of the $\mathrm{BBB}$ permeability of drugs, but the results obtained revealed a low correlation between in vitro and in vivo data. Thus, Caco-2 may not be useful for predictions of drug's transport across the BBB (Lundquist et al., 2002; Garberg et al., 2005). Both compounds were shown to be potential substrates for P-glycoprotein (P-gp) which effluxes drugs and various compounds to undergo further metabolism and clearance (Amin, 2013) resulting in therapeutic failure because the drug concentration would be lower than expected (Levin, 2012). CYP Inhibitory promiscuity for both Bhutkesoside A and Bhutkesoside B was rated low as both compounds were predicted as non-inhibitors for cytochrome $\mathrm{P} 450$ isoforms which catalyze the metabolism of a wide variety xenobiotics and drugs. Fortunately, both compounds did not show any acute toxicity and mutagenic effect with respect to the AMES test data (Table 3).

Table 3: ADMET predictions using AdmetSAR

\begin{tabular}{lcc}
\hline \multicolumn{1}{c}{ ADMET } & \multicolumn{2}{c}{ Lead } \\
\hline Absorption & Bhutkesoside A & Bhutkesoside B \\
\hline BBB & BBB+ & BBB- \\
HIA & HIA- & HIA- \\
Caco-2 & Caco-2- & Caco-2- \\
\hline Distribution \& Metabolism & & Non-substrate \\
\hline CYP450 substrate & Non-substrate & Non-inhibitor \\
CYP450 inhibitor & Non-inhibitor & Low \\
CYP IP & Low & \\
(Inhibitory promiscuity) & & Weak inhibitor \\
Excretion \& Toxicity & & Non-inhibitor \\
\hline HERG & Weak inhibitor & Non toxic \\
Inhibition & Non-inhibitor & Non-carcinogen \\
AMES toxicity & Non toxic & Low \\
Carcinogen & Non-carcinogen & High \\
Fish toxicity & Low & High \\
T.P toxicity & High & Not ready \\
H.B toxicity & High & biodegradable \\
Biodegradation & Ready & Category III \\
Acute Oral Toxicity & biodegradable & Category III
\end{tabular}

Key: BBB: Blood Brain Barrier, HIA: Human Intestinal Absorption, HERG Human Ether-a-go-go-Related Gene, TP: Tetrahymena Pyriformis, HB: Honey Bee, RAT: Rat acute toxicity, FT: Fish toxicity.

MetaPrint2D predictions revealed that Bhutkesoside A has only one site, with high Normalized Occurrence Ratio (NOR), for metabolic transformations, whereas, Bhutkesoside B has three metabolic sites with high NOR (Figure 3). The red colored hydroxyl group of Bhutkesoside $\mathrm{A}$ and the red colored methine carbons and oxygen atom in Bhutkesoside B represent good sites for metabolism. The hydroxyl group of Bhutkesoside A could undergo oxidation, phosphorylation, glucuronidation, dehydroxylation, sulfation, methylation or glucosidation. With regard to Bhutkesoside $\mathrm{B}$, the methine carbon atoms, in the two rings, were predicted to undergo dealkylation only and the resultant hydroxyl groups would undergo phosphorylation. 


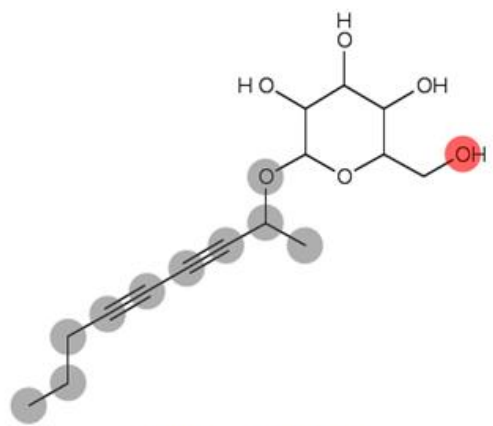

Bhutkesoside A

$\begin{array}{ll}\text { Red } & 0.66<=\text { NOR }<=1.00 \\ \text { Orange } & 0.33<=\text { NOR }<0.66\end{array}$

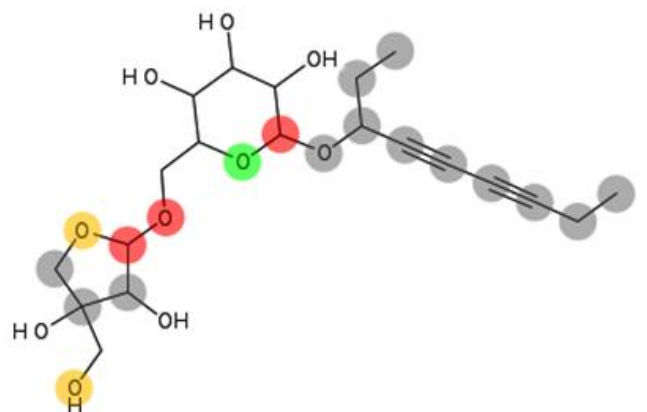

Bhutkesoside B

$\begin{array}{ll}\text { Green } & 0.15<=\text { NOR }<0.33 \\ \text { White } & 0.00<=\text { NOR }<0.15 \\ \text { Grey } & \text { Little/no data }\end{array}$

Fig. 3: Metabolic predictions using Metaprint2D for each lead. The color highlighting an atom indicates its Normalized Occurrence Ratio (NOR). A high NOR indicates a more frequently reported site of metabolism in the metabolite database. Atoms are colored according to the likelihood of a metabolic site; High: red, Medium: orange, Low: green, very low is not colored, and No data: grey.

\section{CONCLUSION}

In silico target prediction and molecular docking revealed that the two diacetylene glycosides Bhutkesoside $\mathrm{A}$ and Bhutkesoside B are potential uridine-cytidine kinase 2 (UCK2) inhibitors. Since UCK2 is over expressed in proliferating cancer cells, the two compounds are herein proposed as new leads for cancer chemotherapy. To further estimate the druggability of the two leads, their physicochemical properties and ADMET profiles have been addressed computationally.

\section{ACKNOWLEDGMENT}

All authors acknowledge Faculty of Pharmacy, University of Khartoum, SUDAN for support and assistance.

Financial support and sponsorship: Amina I. Dirar acknowledges the partial financial support provided by the National University, SUDAN.

Conflict of Interests: There are no conflicts of interest.

\section{REFERENCES}

Adhikari B, Devkota HP, Joshi KR, Watanabe T, Yahara S. Two new diacetylene glycosides: bhutkesoside A and B from the roots of Ligusticopsis wallichiana. Nat Prod Res, 2015; $11: 1-8$.

Amin ML. P-glycoprotein inhibition for optimal drug delivery. Drug Target Insights, 2013; 7:27-34.

Appleby TC, Larson G, Cheney IW, Walker H, Wu JZ, Zhong W, Hong Z, Yao N. Structure of human uridine-cytidine kinase 2 determined by SIRAS using a rotating-anode $\mathrm{X}$-ray generator and a single samarium derivative. Acta Crystallogr D Biol Crystallogr, 2005; 61:278284

Balunas MJ, Kinghorn AD. Drug discovery from medicinal plants. Life Sci, 2005; 78:431-441.

Carlsson L, Spjuth O, Adams S, Glen RC, Boyer S. Use of historic metabolic biotransformation data as a means of anticipating metabolic sites using MetaPrint2D and Bioclipse. BMC Bioinformatics, 2010;11:362. [ONLINE] Available at: http://www.biomedcentral.com/1471-2105/11/362. [Accessed 04 June 2016].
Cheng F, Li W, Zhou Y, Shen J, Wu Z, Liu G, Lee PW, Tang Y. admetSAR: a comprehensive source and free tool for assessment of chemical ADMET properties. J Chem Inf Model, 2012; 52:3099-3105. Connolly GP, Duley JA. Uridine and its nucleotides: Biological actions, therapeutic potentials. Trends Pharmacol Sci, 1999; 20:218-225.

Dirar AI, Waddad AY, Mohamed MA, Mohamed MS, Osman WJ, Mohammed MS, Elbadawi MAA. In silico pharmacokinetics and molecular docking of three leads isolated from Tarconanthus camphoratus L. Int J Pharm Pharm Sci, 2016; 8:71-77.

Earm K, Earm YE. Integrative approach in the era of failing drug discovery and development. Integr Med Res, 2014; 3:211-216.

Garberg P, Ball M, Borg N, Cecchelli R, Fenart L, Hurst RD, Lindmark T, Mabondzo A, Nilsson JE, Raub TJ, Stanimirovic D, Terasaki T, Oberg JO, Osterberg T. In vitro models for the blood-brain barrier. Toxicol In Vitro, 2005; 19:299-334.

Guex N, Peitsch MC. SWISS-MODEL and the SwissPdbViewer: an environment for comparative protein modeling. Electrophoresis, 1997; 18:2714-2723.

Hubatsch I, Lazorova L, Vahlne A, Artursson P. Orally active antiviral tripeptide glycyl-prolyl-glycinamide is activated by CD26 (dipeptidyl peptidase IV) before transport across the intestinal epithelium. Antimicrob Agents Chemother, 2005; 49:1087-1092.

Levin GM. P-glycoprotein: why this drug transporter may be clinically important. Current Psychiatry, 2012;11. [ONLINE] Available at: http://www.currentpsychiatry.com/articles/savvy-psychopharmacology. [Accessed 04 June 2016].

Lipinski CA, Lombardo F, Dominy BW, Feeney PJ. Experimental and computational approaches to estimate solubility and permeability in drug discovery and development settings. Adv Drug Deliv Rev, 2001; 46:03-26.

Liu X, Ouyang S, Yu B, Liu Y, Huang K, Gong J, Zheng S, Li $\mathrm{Z}$, Li H, Jiang H. PharmMapper server: a web server for potential drug target identification using pharmacophore mapping approach. Nucleic Acids Res, 2010; 38:W609-W614.

Lundquist S, Renftel M, Brillault J, Fenart L, Cecchelli R, Dehouck MP. Prediction of drug transport through the blood-brain barrier in vivo: a comparison between two in vitro cell models. Pharm Res, 2002; 19:976-981.

Malami I, Abdul AB, Abdullah R, Bt Kassim NK, Waziri P, Christopher E. In silico discovery of potential uridine-cytidine kinase 2 inhibitors from the rhizome of Alpinia mutica. Molecules, 2016; 21, 417. [ONLINE] Available at: http://www.mdpi.com/1420-3049/21/4/417. [Accessed 04 June 2016].

Mandal S, Moudgil M, Mandal SK. Rational drug design. European Journal of Pharmacology, 2009; 625: 90-100.

Morris GM, Goodsell DS, Halliday RS, Huey R, Hart WE, Belew RK, Olson AJ. Automated docking using a Lamarckian genetic 
algorithm and empirical binding free energy function. J Comput Chem, 1998;19:1639-1662.

Morris GM, Huey R, Lindstrom W, Sanner MF, Belew RK, Goodsell DS, Olson AJ. Autodock4 and AutoDockTools4: automated docking with selective receptor flexiblity, J Comput Chem, 2009; 30:2785-2791.

Murata D, Endo Y, Obata T, Sakamoto K, Syouji Y, Kadohira M, Matsuda A, Sasaki T. A crucial role of uridine/cytidine kinase 2 in antitumor activity of 3'-ethynyl nucleosides. Drug Metab Dispos, 2004;32:1178 - 1182 .

Neamati N, Barchi JJ Jr. New paradigms in drug design and discovery. Curr Top Med Chem, 2002; 2:211-227.

O'Boyle NM, Banck M, James CA, Morley C, Vandermeersch T, Hutchison GR. Open Babel: an open chemical toolbox. Journal of Cheminformatics, 2011; 3:33. [ONLINE] Available at: http://www.jcheminf.com/content/3/1/33. [Accessed 04 June 2016].

Padalia RC, Verma RS, Chauhan A, Chanotiya CS, Yadav A. Variation in the volatile constituents of different plant parts of Ligusticopsis wallichiana from western Himalaya, India. Nat Prod Commun, 2012; 7:1077-1078.

Pettersen EF, Goddard TD, Huang CC, Couch GS, Greenblatt DM, Meng EC, Ferrin TE. UCSF Chimera-a visualization system for exploratory research and analysis. J Comput Chem, 2004; 25:1605-1612.

Qian Y, Ding Q, Li Y, Zou Z, Yan B, Ou L. Phosphorylation of uridine and cytidine by uridine-cytidine kinase. J Biotechnol, 2014; 188:81-87.

Sliwoski G, Kothiwale S, Meiler J, Lowe EW Jr. Computational Methods in Drug Discovery, Pharmacol Rev, 2014; 66: 334-395.

Srimai V, Ramesh M, Parameshwar KS, Parthasarathy T. Computer-aided design of selective cytochrome P450 inhibitors and docking studies of alkyl resorcinol derivatives. Med Chem Res, 2013; 22:5314-5323.

Sripayap P, Nagai T, Uesawa M, Kobayashi H, Tsukahara T, Ohmine K, Muroi K, Ozawa K. Mechanisms of resistance to azacitidine in human leukemia cell lines. Exp Hematol, 2014; 42:294-306
Suzuki NN, Koizumi K, Fukushima M, Matsuda A, Inagaki F. Structural basis for the specificity, catalysis and regulation of human uridine-cytidine kinase. Structure, 2004; 12:751-764.

Valencia A, Masala E, Rossi A, Martino A, Sanna A, Buchi F, Canzian F, Cilloni D, Gaidano V, Voso MT, Kosmider O, Fontenay M, Gozzini A, Bosi A, Santini V. Expression of nucleoside-metabolizing enzymes in myelodysplastic syndromes and modulation of response to azacitidine. Leukemia, 2014; 28:621-628.

van de Waterbeemd $\mathrm{H}$, Gifford E. ADMET in silico modeling: towards prediction paradise?. Nat Rev Drug Discov, 2003; 2:192-204.

Van Rompay AR, Norda A, Lindén K, Johansson M, Karlsson A. Phosphorylation of uridine and cytidine nucleoside analogs by two human uridine-cytidine kinases. Mol Pharmacol, 2001; 59:1181-1186.

Veber DF, Johnson SR, Cheng HY, Smith BR, Ward KW, Kopple KD. Molecular properties that influence the oral bioavailability of drug candidates. J Med Chem, 2002; 45:2615-2623.

Waterhouse RN. Determination of lipophilicity and its use as a predictor of blood-brain barrier penetration of molecular imaging agents. Mol Imaging Biol, 2003; 5:376-389.

Zlatopolskiy BD, Morgenroth A, Kunkel FH, Urusova EA, Dinger C, Kull T, Lepping C, Reske SN. Synthesis and biologic study of IV-14, a new ribonucleoside radiotracer for tumor visualization. J Nucl Med, 2009; 50:1895-1903.

\section{How to cite this article:}

Mohamed MA, Dirar AI, Hamdoun S. Discovery of two diacetylene glycosides as human uridine-cytidine kinase 2 inhibitors: an in silico approach. J App Pharm Sci, 2016; 6 (11): 034-039. 